\title{
Le désengagement du canada dans les AFFAIRES INTERNATIONAUX
}

\author{
Par Julie Roy
}

Abstract

This article analyses the downfall of Canada's participation and influence over international peacekeeping missions in the post-Cold War era. The author begins by discussing how the collapse of the USSR led to fundamental changes in the system of international relations. The author then centres her discussion on Canada, focusing primarily on the national and international considerations that are influencing the nation's involvement in missions overseas.

\section{Introduction}

Selon certains analystes de la politique étrangère canadienne, nous assistons présentement au désengagement du Canada dans les affaires internationales ${ }^{1}$. Sans rejeter complètement la thèse d'un retour à l'isolationnisme, il est à noter qu'en dépit du changement remarquable de la conjoncture internationale depuis la fin de la guerre froide et des soubresauts de sa politique interne, le Canada a su maintenir de façon appréciable son engagement multilatéral. D'ailleurs, l'implication impressionnante du Canada dans les opérations de maintien paix trahit en quelque sorte cette nouvelle tendance au repli prêtée au gouvernement canadien. 
L'apport du Canada aux opérations de maintien de la paix est irréfutable. Parmi ses faits d'armes, il y a la proposition de la mise sur pied des désormais célèbres Casques bleus. Une proposition qui vaudra à son instigateur, le ministre des Affaires étrangères canadien M. Lester B. Pearson, le prix Nobel de la paix en 1957 et au Canada, la reconnaissance de son influence diplomatique spécialement dans le domaine du maintien de la paix. La constance de l'engagement canadien en matière de paix et sécurité contribua également à procurer au Canada une expertise fort enviable en tant que gardien de la paix. De fait, le Canada a participé à toutes les missions de maintien de la paix dirigées par les Nations Unies jusqu'en 1988 et s'implique toujours dans une quantité considérable d'opérations. La redéfinition du contexte mondial et le repositionnement des acteurs internationaux au lendemain de la guerre froide ont toutefois fait évoluer considérablement l'exercice du maintien de la paix. Malgré la solide réputation que le Canada s'est bâtie au cours des années, ses ressources ne lui permettent plus d'intervenir dans des conflits plus nombreux et plus intenses. Le Canada doit dorénavant faire des choix. Ces choix sont-ils motivés par une stratégie ficelée et bien définie par les dirigeants canadiens ? Absolument. Et c'est ce que je tenterai de démontrer dans le présent travail.

$\mathrm{Au}$ cours des pages qui suivront, je présenterai les considérations nationales et internationales qui à mon sens, influencent directement les autorités canadiennes à intervenir ou pas dans une opération pour la paix. D'abord, je dresserai une liste des conditions générales à la participation canadienne dans une telle opération pour ensuite enchaîner avec des conditions spécifiques. À leur nombre, il y a la sécurisation des intérêts économiques, le jeu des alliances et la poursuite de l'engagement multilatéral et enfin, le maintien de l'influence $\mathrm{du}$ Canada sur la scène internationale et le développement d'un nouveau leadership. Toutefois, avant de nous lancer dans le vif du sujet, j'estime important d'effectuer une brève mise en contexte de la nouvelle conjoncture internationale à l'intérieur duquelle le Canada doit œuvrer ainsi que de rappeler les mutations qu'a subi le concept de maintien de la paix depuis la fin de la guerre froide.

\section{Le Canada et le maintien de la paix dans le contexte d’après guerre froide}


La fin de l'antagonisme est-ouest correspond à l'émergence de deux tendances qui affecteront profondément l'avenir du maintien de la paix. D'abord, il y a l'attitude changeante des États face au maintien de la paix en raison de la modification des impératifs de leur politique extérieure ${ }^{2}$. Le nouveau système unipolaire favorise la globalisation économique ainsi que la mise en place de structure d'échanges et de défense qui exacerbera l'interdépendance entre les États. La sécurité commune est fragilisée par divers phénomènes tels la globalisation, le développement technologique, la prolifération des armements de destruction massive, l'élargissement des ramifications des réseaux criminels et terroristes, etc... Cette nouvelle réalité commande une recrudescence de la vigilance étatique ainsi qu'une coopération et une coordination accrue entre les mécanismes nationaux de défense. Conséquemment, chaque État se sent davantage concerné par les questions de paix et sécurité et envisage avec une ouverture nouvelle leur participation aux opérations de maintien de la paix ${ }^{3}$.

Outre l'engagement plus sérieux d'un plus grand nombre d'États dans les opérations de maintien de la paix, l'augmentation du nombre de conflits devenus pluridimensionnels a beaucoup complexifié les missions sous auspices onusiens ${ }^{4}$. Boutros-Ghali explique comme suit la montée des périls depuis la fin de la guerre froide :

...le sentiment national et la revendication de souveraineté s'affirment ici et là avec une vigueur renouvelée, et la cohésion des États est mise à mal par de violentes luttes ethniques, religieuses, sociales, culturelles ou linguistiques. La paix sociale est menacée, d'une part, par la résurgence des discriminations et des exclusions et, de l'autre, par des actes de terrorisme qui visent à miner la voie démocratique de l'évolution et du changement ${ }^{5}$. (4-para 11).

Les causes des nouveaux conflits sont profondes et nécessitent des interventions mieux rôdées. Les missions de maintien de la paix sont plus dangereuses, plus coûteuses et exigent une action multidimensionnelle déployée tant par les civils que par les militaires. Ces nouvelles caractéristiques du rôle de gardien de la paix sont répertoriées dans l'Agenda pour la paix de 1992. Par cet ouvrage, Boutros-Ghali procède à la segmentation des nouveaux impératifs en matière de maintien de la paix. L'ancien secrétaire général identifie quatre domaines d'intervention distincts pour les Nations Unies soit, la diplomatie préventive, le maintien et le rétablissement de la paix et la consolidation de la paix, une notion qu'il a lui-même introduite ${ }^{6}$. Cette étude qui présente aussi des recommandations sur les moyens de renforcer la capacité de l'organisation dans ces différents domaines, constituera le livre de référence dans la direction des nouvelles 
opérations pour la paix.

Alors qu'au cours de la guerre froide le Canada a su acquérir une influence remarquable notamment en sa qualité de champion du maintien de la paix et de médiateur aguerri ${ }^{7}$, la nouvelle conjoncture internationale et les transformations des opérations de maintien de la paix lui prête peu d'occasion de se démarquer.

For the first time Canada faces enormous competition from other countries that inevitably will challenge the idea of Canada's having a special international vocation as peacekeeper. Just like the Canadian Armed Forces, other national militaries have been freed from the exigencies of East-West competition. They have begun to find or be assigned new roles in the recent explosion in the number of peacekeeping operations $^{8}$.

Les États du tiers-monde s'impliquent davantage dans les opérations de maintien de la paix, parfois en fournissant des contingents de Casques bleus très imposants. Quant aux grandes puissances, elles s'intéressent de plus près à ce type de missions et tendent à y exercer un leadership et une influence tout à fait remarquable 9 .

Alors que la prédominance du Canada en matière de maintien de la paix est défiée de toute part et que son influence en tant que puissance moyenne s'effrite peu à peu ${ }^{10}$, deux éléments importants de sa politique interne détournent son attention de la scène internationale. Il y a d'abord la crise financière publique et les préoccupations pour diminuer le déficit qui mèneront à des coupures drastiques dans le budget de la défense et ensuite, les problèmes constitutionnels qui se traduiront par une montée des valeurs conservatrices au pays ${ }^{11}$. Ces contraintes financières et idéologiques empêcheront la mobilisation des ressources financières et techniques nécessaires au Canada pour demeurer un chef de file dans le domaine de maintien de la paix. Aujourd'hui, le Canada figure au $34^{\mathrm{e}}$ rang des fournisseurs d'effectifs et d'observateurs militaires à la disposition de l'ONU ${ }^{12}$.

Bien que le Canada ne s'affiche plus comme le leader incontesté dans le domaine du maintien de la paix, sa participation demeure importante. Son engagement dans ce type d'activité demeure un aspect primordial de sa politique étrangère et lui permettra peut-être éventuellement de regagner quelques échelons dans la hiérarchie internationale. Ne pouvant plus participer à toutes les 
opérations de maintien de la paix, le gouvernement canadien se doit maintenant d'être plus sélectif. Il se devra de cibler des missions compatibles avec les intérêts et les aspirations nationales.

\section{La stratégie Canadienne face aux opérations de maintien de la paix}

Après consultation des autres ministères fédéraux concernés par les opérations de maintien de la paix dont l'ACDI, le Ministère de la Défense et la Gendarmerie Royale, le Ministère des Affaires étrangères joue un rôle crucial dans l'évaluation des mandats proposés au Canada ${ }^{13}$. Avant de décider de la participation canadienne à une telle opération, certaines conditions sont soigneusement examinées par le MAECI :

- s'il existe un mandat clair et réalisable, conféré par une autorité politique compétente telle le Conseil de sécurité;

- si les parties en conflit ont accepté la présence d'une opération de maintien de la paix de l'ONU et la participation de personnel canadien à cette opération;

- si l'opération de maintien de la paix a lieu dans le contexte d'un processus visant le règlement politique du conflit;

- si l'effectif et la composition internationale de l'opération sont assortis au mandat;

- si l'opération est suffisamment financée et est dotée d'une structure logistique satisfaisante;

- si les risques courus par les Canadiens sont raisonnables;

- si la contribution canadienne serait compatible avec les relations politiques générales entre le Canada et le pays ou la région concernée 
- si le Canada a la capacité de participer à l'opération, compte tenu des engagements multilatéraux qu'il a déjà contractés ${ }^{14}$.

Il en revient toutefois au Cabinet d'approuver définitivement la participation du Canada à une telle mission ${ }^{15}$.

Outre ces quelques considérations d'ordre général, le Canada, à l'instar des autres États, poursuit par l'entremise de son implication dans le processus de maintien de la paix un certain nombre d'intérêts stratégiques. Chaque conflit ou opportunité d'intervention est étudié minutieusement et est soumis à un calcul coûts-bénéfices. Cet examen prend en considération les répercussions d'une éventuelle participation entre autres sur « la dynamique des forces politiques internes, sur les relations interalliées, sur la position relative de l'État à l'échelle régionale et internationale, sur les mouvement de réfugiés ou d'autres menaces directes à la sécurité nationale, par rapport au prestige qu'elles peuvent générer ${ }^{16} . \gg$ Observons de plus près les intérêts du Canada dans cet aspect précis de sa politique étrangère.

\section{La sécurisation des intérêts économiques}

En raison de son isolement, le territoire canadien est relativement bien protégé de toute menace externe à sa sécurité. D'ailleurs, depuis la fin de la guerre froide, les Canadiens bénéficient d'un climat de sécurité tout à fait enviable. Si les nombreux conflits faisant rage à l'échelle du globe ont jusqu'ici peu affecté la sécurité des Canadiens, les intérêts économiques se montrent beaucoup plus sensibles à pareil tumulte.

À la lumière des événements qui ont marqué la politique canadienne ces dernières années, le gouvernement libéral a fait le choix de prioriser ses intérêts économiques en orientant sa politique étrangère vers la promotion du commerce $^{17}$. Un point de vue partagé par Irving Brecher qui atteste l'énorme dépendance du Canada face au commerce international et par conséquent, le dynamisme avec lequel le Canada cherche à conquérir de nouveaux marchés pour l'exportation de ses produits ${ }^{18}$.

Dans son évaluation des mandats proposés dans le cadre d'intervention 
pour le maintien de la paix, le gouvernement canadien prend évidemment en considération l'aspect stratégique que revêtent les régions affectées et les conséquences que le déclenchement ou l'aggravation d'un conflit pourraient avoir sur ses intérêts économiques. C'est ainsi que la sécurisation des marchés conquis ou convoités par le Canada constitue un incitatif considérable dans la décision d'intervenir ou non. Prenons l'exemple de l'Asie et de l'Amérique Latine où le gouvernement canadien tente des percées commerciales par le renforcement de ses relations bilatérales (Japon, Chili, Argentine, Brésil) et multilatérales (APEC, ZLEA).

Canada is showing a new interest in the countries of the Far East and those of Latin America. Prime Minister Jean Chrétien most publicized trade missions have been to theses two areas. Ottawa believes that as Canada seeks out new trading opportunities, there should be some commensurate augmentation in military links with regions and countries outside the traditional North Atlantic triangle ${ }^{19}$.

Dans l'optique où Ottawa tente de renforcer ses relations de défense avec les régions d'Asie-Pacifique et d'Amérique Latine pour protéger ses intérêts commerciaux, il n'est pas surprenant de constater son implication grandissante dans ces régions au niveau des opérations de maintien de la paix. En AsiePacifique, le Canada a déployé un large contingent militaire pour soutenir INTERFET au Timor Oriental en $1999^{20}$. Bien que le déploiement se voulait de courte durée (6 mois), il demeure l'un des plus importants efforts militaires du Canada au cours des dernières années ${ }^{21}$. Dans la même région, notons également l'apport considérable des Canadiens dans les opérations au Cambodge depuis 1991 (MIPRENUC et APRONUC) ${ }^{22}$. Du côté de l'Amérique Latine, le Canada y a assumé une présence quasi ininterrompue depuis le début des années 90. Parmi les opérations les plus importantes auxquelles il a participé, il y a celles en Amérique Centrale (ONUCA, 1989-1992), à El Salvador (ONUSAL, 1991-95) et au Guatemala (MINUGUA, 1994-à ce jour) ${ }^{23}$. C'est sans doute en Haïti que l'expertise canadienne en matière de maintien de la paix a été le mieux exploitée via les opérations de l'ONUVEH (1990-91), de la MINUHA (1993-96), de la MANUH (1996-97), de la MITNUH (1997) et de la MIPONUH (1997- à ce jour $)^{24}$. En contrepartie, la participation canadienne aux missions de maintien de la paix en Afrique a été plutôt décevante depuis les désastreuses campagnes en Somalie (1992-93) et au Rwanda (1993-96) ${ }^{25}$. « For the most, Canada does not have major trading or other national interest at stake in Africa, and so its attention to the political crises and conflicts on the continent is sporadic and occasioned by specific circumstances ${ }^{26}$.» Le niveau de dangerosité élevé des opérations constitue sans doute un incitatif supplémentaire dans la décision de 
maintenir les contingents militaires canadiens éloignés du continent africain. Ainsi, le gouvernement canadien participe aux efforts de maintien de la paix en Afrique en fournissant essentiellement une assistance financière au programme de consolidation de la paix et un nombre restreint de polices civiles ${ }^{27}$.

Considérant l'implication canadienne dans les opérations en Afrique, la thèse d'Albrecht Schnabel se vérifie à savoir que « lorsqu'on considère le coût du maintien de la paix dans des régions qui n'ont aucune importance stratégique, l'égoïsme étatique l'emporte... ${ }^{28}{ }^{\prime \prime}$

\section{Le jeu des alliances et l'engagement multilatéral}

Le multilatéralisme a toujours été pour le Canada un moyen privilégié pour 1'atteinte des objectifs de sa politique étrangère ${ }^{29}$. Tout au long de la guerre froide, le Canada a vigoureusement défendu les régimes multilatéraux et les institutions internationales, deux types de dispositifs particulièrement favorables à l'ouverture et à la vulnérabilité de son économie ${ }^{30}$. Cette spécificité de la politique étrangère du Canada a beaucoup contribué au développement de son statut de puissance moyenne et à l'acquisition d'une influence importante à l'intérieur des instances internationales ${ }^{31}$.

Ce profond attachement au multilatéralisme incita le Canada au fil des ans, à adhérer à une quantité impressionnante d'organisations internationales. Toutefois, son engagement auprès des Nations Unies est sans contredit le plus précieux de tous. De tout temps, l'ONU est apparue comme « l'une des pierres angulaires de la politique de sécurité du Canada ${ }^{32} »$. La place privilégiée qu'occupe l'organisation internationale dans la politique étrangère canadienne explique la contribution exceptionnelle d'Ottawa dans les opérations de maintien de la paix dirigées par l'ONU. De 1956 à 1988, le Canada fut « le premier pourvoyeur de Casques bleus et le recordman de la participation aux 13 missions alors mises sur pied par les Nations Unies ${ }^{33} \gg$. Par la suite, en raison de l'augmentation significative du nombre d'opérations et de l'émergence de problèmes nationaux importants, le Canada dû se contenter d'une participation plus limitée. Entre 1988 et 1997, il est intervenu dans 15 des 32 missions mises en place par les Nations Unies ${ }^{34}$.

Aujourd'hui, la tendance à la rationalisation des effectifs militaires canadiens mis à la disposition des Nations Unies pour les opérations de maintien de la paix s'est maintenue. Le gouvernement canadien tente d'appuyer un 
maximum de missions en y apportant une contribution qui, à l'instar des ressources financières nationales, s'avère très modeste. Cette stratégie vise à démontrer la solidarité du Canada mais surtout à maintenir sa présence et à sauvegarder, autant que faire se peut, une certaine influence à l'intérieur de l'organisation multinationale ${ }^{35}$. Ainsi, les décideurs canadiens choisissent fréquemment de soutenir les opérations de maintien de la paix de l'ONU mais ce soutien est souvent réduit à l'envoi d'un nombre restreint d'observateurs ou d'officiers civils.

Si le Canada se contente d'une place moins prédominante dans les opérations de maintien de la paix dirigées par les Nations Unies, c'est que ses priorités ont changé. Dans l'élan de la réforme onusienne, une solution non négligeable fut apportée dans l'ordre de partager le fardeau du maintien de la paix. Cette solution est de décentraliser le commandement des opérations à différentes organisations régionales telles l'OEA, le CSCE et l'OTAN ${ }^{36}$. Le semi retrait du Canada dans les missions dirigées par l'ONU s'explique largement par l'entrée en force de l'OTAN dans le domaine du maintien de la paix.

En étudiant les opérations à l'intérieur desquelles le Canada s'est militairement le plus investi dernièrement, on constate que la plupart sont dirigées par l'OTAN. Ce critère primordial de la stratégie canadienne quant à la participation aux missions de maintien de la paix est compréhensible à plus d'un égard. D'abord, en fermant ses deux bases européennes et en réduisant drastiquement ses forces armées sur le vieux continent en 1991, le Canada diminua considérablement sa présence militaire au sein de l'OTAN. De plus, cette manœuvre contribua à lever les doutes de ses partenaires de l'Alliance Atlantique quant au sérieux de son engagement dans la défense de la sécurité européenne et à affaiblir la légitimité d'Ottawa sur les questions relatives à l'Europe ${ }^{37}$. Ensuite, en raison des élargissements de l'OTAN, l'organisation tend à s'européaniser ${ }^{38}$. Par conséquent, le Canada fait face à une compétition accrue dans l'exercice de son influence. Ces réalités expliquent la nécessité pour le Canada de s'investir intensivement dans les initiatives de l'Alliance Atlantique afin de s'imposer comme un membre actif, fiable et sérieux ${ }^{39}$.

Considérant l'importance que des institutions telles l'OTAN et l'ONU revêtent pour les intérêts stratégiques canadiens ${ }^{40}$, l'implication militaire massive du Canada dans le conflit en ex-Yougoslavie depuis 1991 obéit donc à une logique indéniable. «Si le Canada n'a que peu d'intérêts dans les Balkans, il en a 
au niveau de la sécurité européenne ${ }^{41}$ et des institutions multilatérales telles l'ONU et l'OTAN. Ainsi, de 1992 à 1996 des milliers de Casques bleus canadiens ont renfloué les rangs de la FORPRONU, opération dirigée par l'ONU en ex-Yougoslavie. La participation militaire canadienne à cette opération fut la cinquième en importance ${ }^{42}$. L'appui aux initiatives de l'OTAN dans la région fut tout aussi considérable. En effet, près de 1083 soldats ont participé à l'opération IFOR en Bosnie-Herzégovine et 1269 soldats prennent toujours part à celle qui lui succède, en l'occurrence, l'opération $\mathrm{SFOR}^{43}$.

Enfin, le Canada manifeste un appui hors normes aux forces de l'OTAN dans leur campagne en Afghanistan. Dans le cadre de l'opération Athena, environ 1900 soldats sont affectés lors de chaque rotation ce qui fait du contingent canadien, le plus imposant de la force internationale d'assistance à la sécurité $^{44}$. Ceci constitue l'un des plus vastes efforts militaires fourni par le Canada au cours des dernières années. Aussi, notons la présence d'un imposant contingent militaire canadien dans la Force multinationale intérimaire débarquée à Haïti en mars dernier. Près de 450 soldats y ont été affectés par le Canada ${ }^{45}$.

\section{Le maintien du statut de puissance moyenne et définition d'un nouveau leadership}

Malgré une volonté évidente de participer activement aux initiatives de l'ONU et de l'OTAN en matière de maintien de la paix, le Canada connaît présentement une perte d'influence indéniable dans un domaine où autrefois, il était un chef de file incontesté. La présence accentuée des grandes puissances dans le domaine du maintien de la paix diminue la nécessité, pour la communauté internationale, de recourir aux services de médiateurs ou d'honnêtes courtiers comme ceux qu'offraient les puissances moyennes, telle le Canada, pendant de la guerre froide ${ }^{46}$. Alors que le statut de puissance moyenne du Canada est remis en question, sa politique étrangère a subi, depuis la fin de la guerre froide, deux changements notoires. Ces modifications consistent d'abord à l'élaboration de la doctrine de sécurité humaine et ensuite, au déplacement des priorités du maintien de la paix vers la consolidation de la paix ${ }^{47}$. Ce sont sur ces deux nouvelles facettes de sa politique étrangère, qu'Ottawa tente aujourd'hui de capitaliser par le biais de sa participation aux opérations de maintien de la paix. De fait, dans sa stratégie, le gouvernement canadien priorise les mandats faisant appel aux compétences nationales en matière de sécurité humaine et de consolidation de la paix et ce, dans l'optique de redéfinir un nouveau leadership 
sur lequel l'influence du Canada pourrait s'appuyer.

La sécurité humaine est un concept mis de l'avant par Lloyd Axworthy lors de la définition de la nouvelle politique étrangère canadienne en 1994. Dans un article publié par The International Journal, l'ancien ministre des Affaires étrangères canadiens définit la doctrine comme suit :

\begin{abstract}
human security is much more than the absence of military threat. It includes security against economic privation, an acceptable quality of life, and a guarantee of fundamental human rights. This concept of human security recognizes the complexity of the human environment and accepts that the forces influencing human security are interrelated and mutually reinforcing. At a minimum, human security requires that basic needs are met, but it also acknowledges that sustained economic development, human rights and fundamental freedom, the rule of low, good governance, sustainable development and social equity are as important to global peace as arms control and disarmaments ${ }^{48}$.
\end{abstract}

Le Canada milite farouchement pour la promotion de la sécurité humaine auprès des instances onusiennes. Ces pressions exercées par les dirigeants canadiens visent à convaincre les membres de l'organisation multilatérale de la nécessité de procurer à la sécurité humaine une place de choix dans l'agenda mais surtout dans la réforme imminente des Nations Unies.

Afin d'appuyer ses efforts diplomatiques pour mousser le concept de sécurité humaine, le Canada aspire à prendre l'initiative de sa cause sur le terrain. En effet, dans sa décision de soutenir les efforts pour le rétablissement de la paix, « le Canada examine attentivement toute demande d'intervention dont le but est la promotion de la sécurité humaine ${ }^{49} . » D^{\prime}$ 'ailleurs, plusieurs interventions canadiennes au cours des dernières années, notamment en Afrique, ont été motivées par cette préoccupation. Effectivement, l'engagement canadien dans les opérations en Sierra Leone (UNAMSIL) et en République démocratique du Congo (MONUC) fut décidé en raison de la convergence entre les mandats proposés et les intérêts nationaux canadiens à l'égard des conflits à caractère humanitaire relevant de la sécurité humaine ${ }^{50}$.

Le deuxième champ ciblé par Ottawa pour le développement d'une nouvelle expertise canadienne et pour l'affirmation d'un nouveau leadership est la consolidation de la paix. " Theoretically, peacebuilding is designed to respond to the more complex nature of today's missions in war-torn society. 
Peacebuilding is intended to constitute a more pro-active strategy that address the root causes of conflict, rather than reacting to the immediate crisis ${ }^{51}$.» Les efforts du Canada dans ce domaine s'étendent de la participation à l'édification d'institutions démocratiques, au développement de la société civile et de la coopération inter-communale, à la promotion de la stabilité à l'intérieur de communautés ethniquement divisées, à l'encouragement d'un leadership modéré ainsi qu'à la protection et à la promotion des droits des femmes et des enfants ${ }^{52}$.

Les stratèges nationaux qui décident des interventions canadiennes pour le maintien de la paix considèrent donc avec grand sérieux la nature de l'aide demandée et priorisent les mandats qui sauront mettre en valeur les capacités particulières du Canada. Ainsi, le choix d'appuyer la mission de l'ONU en République centrafricaine (1998-99) s'explique par la mise en évidence d'une unité de communication canadienne spécialisée alors que celui de s'impliquer au Mozambique (1992-94) fut motivé par le recours aux compétences canadiennes en matière de déminage ${ }^{53}$. L'expertise du Canada est dominante dans certains champs spécifiques de la consolidation de la paix tels le déminage et les campagnes d'interdiction des mines antipersonnelles, la protection des femmes et des enfants de la guerre, les télécommunications, la formation aux officiers civils, et le contrôle des armes légères ${ }^{54}$. Le Canada a pu mettre à profit ces compétences particulières en acceptant de participer à certaines missions de consolidation de la paix. Au nombre de ces missions nous comptons celles à Haïti (1994-96), en Bosnie Herzégovine (1996-97), en Afrique occidentale (1998), au Cambodge (1992- à ce jour), dans la région des Grands Lacs africains (1996) ainsi qu'au Guatemala (depuis 1996) ${ }^{55}$.

À l'instar du concept de sécurité humaine, le Canada procède à une énergique promotion de la consolidation de la paix dans l'enceinte des Nations Unies. Dernièrement, le Canada s'est particulièrement affairé à exposer la nécessité, pour les Nations Unies, de se munir d'un dispositif de réaction rapide $(\text { REACT })^{56}$. Malgré l'échec du projet de Rapid Deployable Mission Headquarter (RDMHQ), les efforts du Canada pour promouvoir la mise en place d'un mécanisme de réaction rapide ne furent pas laissés vain. En effet, le Canada participa à la l'édification d'une brigade prête à intervenir en état d'alerte avancée, la SHIRBRIG (Stand-by Forces High Readiness Brigade) qui sera mise à la disposition de 1'ONU en $1999^{57}$. En 2000, le Canada poursuit la logique selon laquelle il s'implique dans des opérations mettant en évidence ses compétences propres et appuyant les causes qu'il défend aux Nations Unies. C'est ainsi qu'il prit part à la première mission de la SHIRBRIG qui se déroula 
en Éthiopie/Érythrée en novembre $2000^{58}$.

En somme, en identifiant la sécurité humaine comme son nouveau cheval de bataille et la consolidation de la paix comme son champ d'action privilégié, le Canada a su définir un créneau qui spécialisera son apport au maintien de la paix. Par conséquent, afin de rendre cohérente sa démarche et d'acquérir une certaine réputation auprès de ses pairs en ces domaines, le Canada cherchera à participer à des opérations qui contribueront à développer et à affirmer ses nouvelles compétences particulières. Cette manœuvre, appuyée par une vaste campagne de promotion auprès des Nations Unies, vise à maintenir l'influence du Canada dans le domaine du maintien de la paix et par le fait même, à lui préserver un statut enviable sur l'échiquier international.

\section{Conclusion}

L'implication du Canada dans les opérations de maintien de la paix répond de toute évidence à une stratégie définie par des intérêts canadiens spécifiques. D'abord, la sécurisation des zones d'échange où le Canada est particulièrement actif ou projette de le devenir, est sans conteste un critère de première importance dans le choix des décideurs canadiens de participer ou non à une opération de maintien de la paix. Tel que démontré auparavant, Ottawa fait montre d'une plus grande volonté à intervenir dans un conflit risquant d'avoir des répercussions directes sur les intérêts économiques canadiens. Ceci explique en partie la forte présence canadienne dans les opérations de maintien de la paix se déroulant en Amérique Latine ainsi que la visibilité accrue de nos Casques bleus dans les missions en Asie.

En second lieu, la stratégie canadienne en matière de maintien de la paix obéit à la nécessité pour le Canada de poursuivre son engagement multilatéral traditionnel. L'ONU et l'OTAN constituent les deux plus importants organismes de sécurité auxquels appartient le Canada ${ }^{59}$. Ces instances sont d'autant plus indispensables au gouvernement canadien puisqu'elles lui procurent une voix dans les mécanismes multilatéraux ainsi qu'une tribune internationale pour la promotion de ses intérêts nationaux. Ainsi, la participation du Canada aux opérations maintien de la paix de l'ONU et de l'OTAN est importante dans la 
mesure où elle lui permet de réaffirmer sa présence internationale de même que son engagement auprès de ces deux institutions. Tel que mentionné plus tôt, la tendance du gouvernement canadien est, à l'heure actuelle, de soutenir massivement les opérations dirigées par l'OTAN, une institution à l'intérieur duquelle son influence est contestée par l'arrivée de nouveaux membres européens. D'autre part, le poids du Canada à l'ONU dans le secteur du maintien de la paix n'est plus ce qu'il était autrefois. De fait, une trentaine de pays déclassent aujourd'hui le Canada au chapitre des fournisseurs d'effectifs et d'observateurs militaires. Ainsi, dans le cadre des opérations conduites par les Nations Unies, le Canada se contente de participer en apportant une contribution minimale dans l'ordre de rappeler sa présence et son soutien à l'organisation multinationale.

Enfin, le Canada choisit de participer à des missions qui lui permettront peut-être d'affirmer son leadership dans les domaines de la sécurité humaine et de la consolidation de la paix. En ciblant des conflits lui procurant l'opportunité de faire démonstration de ses compétences particulières, le Canada poursuit l'objectif de mettre en œuvre une expertise canadienne spécialisée et reconnue. L'établissement de cette expertise vise à accroître la visibilité du Canada au plan international et éventuellement, à faire de lui un joueur essentiel dans ces deux nouveaux aspects du maintien la paix.

Que doit-on penser de cette stratégie canadienne? D'abord qu'elle est mue part certains impératifs qui ne contribueront probablement pas à réaffirmer l'indispensabilité du Canada en matière de maintien de la paix. J'entends évidemment ici la priorité accordée aux missions visant à sécuriser les intérêts économiques canadiens ainsi qu'à sauvegarder l'influence du Canada auprès de l'ONU et de l'OTAN, et ce, particulièrement dans le domaine du maintien de la paix. À l'égard de ce dernier motif, notons que la position du Canada est très clairement fragilisée par une concurrence toujours plus féroce et par des ressources nationales toujours plus pauvres. Si le poids du Canada a considérablement diminué aux Nations Unies quant au maintien de la paix, il se pourrait que ce ne soit qu'une question de temps avant que pareil phénomène soit observable à l'OTAN.

Dans un deuxième temps, de la stratégie étudiée nous devons retenir l'ingéniosité d'une spécialisation canadienne dans le désormais très vaste domaine du maintien de la paix. Alors que le concept de sécurité humaine est 
probablement trop vague pour être appliqué systématiquement et pour récolter ses fruits (Dawson, 313), il en est autrement du concept de consolidation de la paix. Considérant les modestes moyens des portefeuilles canadiens ainsi que les valeurs, l'expertise et l'enviable réputation qu'a développé le Canada en tant que puissance moyenne durant la guerre froide, le domaine de la consolidation de la paix semble tout indiqué pour le développement d'un nouveau leadership canadien. De plus, cette nouvelle approche du maintien de la paix permettrait au Canada de se distinguer des autres États plus fortunés et résolument engagés dans le processus de maintien ou de rétablissement de la paix. En somme, j'appuie les propos de Charles-Philippe David et de Martin Bourgeois à savoir qu'en faisant de la consolidation de la paix le nouveau créneau de la politique étrangère canadienne, le Canada maximiserait ses chances de se tailler une fois de plus, une place de choix parmi les plus influents de ce monde.

\section{ENDNOTES}

${ }^{1}$ Rioux et Hay 24.

${ }^{2}$ Schnabel 201.

${ }^{3}$ Boutros-Gahli 8.

${ }^{4}$ David et Roussel 55.

${ }^{5}$ Boutros-Gahli 11.

${ }^{6}$ Ibid. 1 et 5 .

${ }^{7}$ Donneur et Roussel 143.

${ }^{8}$ Jockel 23.

${ }^{9}$ Donneur et al. 147.

${ }^{10}$ David et al. 41.

${ }^{11}$ Rioux et al. 16. 
${ }^{12}$ Canada. Politique de défense. $<$ http://www.forces.gc.ca/admpol/fra/defence/ peace_supops_f.htm>.

${ }^{13}$ Canada. Le Canada et le maintien de la paix. <http://www.dfait-maeci.gc.ca/ peacekeeping/actors-fr.asp $>$.

${ }^{14}$ Ibid. < http://www.dfait-maeci.gc.ca/peacekeeping/actors-fr.asp >.

${ }^{15}$ Ibid. <http://www.dfait-maeci.gc.ca/peacekeeping/actors-fr.asp >.

${ }^{16}$ Schabel 207.

${ }^{17}$ Sokolsky 39.

${ }^{18}$ Brecher 9.

${ }^{19}$ Sokolsky 39.

${ }^{20}$ Canada. Politique de défense. <http://www.forces.gc.ca/admpol/fra/defence/ peace_supops_f.htm>.

${ }^{21}$ Dawson 304.

${ }^{22}$ Canada. Politique de défense. <http://www.forces.gc.ca/admpol/fra/defence/ peace_supops_f.htm>.

${ }^{23}$ Canada. Politique de défense. < http://www.forces.gc.ca/admpol/fra/defence/ peace_supops_f.htm>.

${ }^{24}$ Canada. Politique de défense. <http://www.forces.gc.ca/admpol/fra/defence/ peace_supops_f.htm>.

${ }^{25}$ Canada. 1994 Livre blanc. <http://www.forces.gc.ca/admpol/fra/ doc/5118_f.htm>.

${ }^{26}$ Dawson 306.

${ }^{27}$ Dawson 306.

${ }^{28}$ Schnabel 211. 
${ }^{29}$ Sokolsky 39.

${ }^{30}$ David et al. 44-45.

${ }^{31}$ Donneur et al. 143.

${ }^{32}$ Ibid. 146.

${ }^{33}$ Coulon 110.

${ }^{34}$ Coulon 110.

${ }^{35}$ Jockel 14.

${ }^{36}$ Ibid. 8.

${ }^{37}$ Donneur 145.

${ }^{38}$ Canada. 1994 Livre blanc. <http://www.forces.gc.ca/admpol/fra/ doc/5118_f.htm>.

${ }^{39}$ Ibid. <http://www.forces.gc.ca/admpol/fra/doc/5118_f.htm>.

${ }^{40}$ Ibid. <http://www.forces.gc.ca/admpol/fra/doc/5118_f.htm>.

${ }^{41}$ Donneur et al. 144.

${ }^{42}$ Ibid. 150.

${ }^{43}$ Canada. Le Canada et le maintien de la paix. <http://www.dfait-maeci.gc.ca/ peacekeeping/missions-fr.asp $>$.

${ }^{44}$ Canada. Opérations courantes. $<$ http://www.forces.gc.ca/site/operations/ Athena/index_f.asp $>$.

${ }^{45}$ Ibid. <http://www.forces.gc.ca/site/operations/Halo/index_f.asp $>$.

${ }^{46}$ David et al. 55 .

${ }^{47}$ Jacoby 185. 
${ }^{48}$ Axworthy 184.

${ }^{49}$ Canada. Le Canada et le maintien de la paix. < http://www.dfait-maeci.gc.ca/ peacekeeping/actors-fr.asp $>$.

${ }^{50}$ Dawson 307.

${ }^{51}$ Jacoby 86.

${ }^{52}$ Ibid. 86.

${ }^{53}$ Canada. Le Canada et le maintien de la paix. < http://www.dfait-maeci.gc.ca/ peacekeeping/actors-fr.asp $>$.

${ }^{54}$ Ibid. <http://www.dfait-maeci.gc.ca/peacekeeping/actors-fr.asp > .

${ }^{55}$ David et al. 589-593.

${ }^{56}$ Canada. Le Canada et le maintien de la paix. < http://www.dfait-maeci.gc.ca/ peacekeeping/actors-fr.asp $>$.

${ }^{57}$ Coulon 114.

${ }^{58}$ Dawson 311.

59 Canada. 1994 Livre blanc. <http://www.forces.gc.ca/admpol/fra/ doc/5118_f.htm>.

\section{Biblographie}

Axworthy, Lloyd. "Canada and human security: the need for leadership." International Journal 52.2 (printemps 1997): 183-196.

Boutros-Ghali, Boutros. Agenda pour la paix. New York: Organisation des Nations Unies, A/47/277-S/24/111, 17 juin 1992. 
Brescher, Irving. "Canadian Foreign Policy: Show me the Money." Behind the Headlines 55.1 (automne 1997): 4-11.

Coulon, Jocelyn. "La politique de défense et le maintien de la paix. Le Canada peut-il faire plus? Et comment?" International Journal 54.1 (1998-1999): 106119.

David, Charles-Philippe et Martin Bourgeois. "Le Canada et la consolidation de la paix. La formulation d'une nouvelle approche pour la politique étrangère canadienne." Études Internationales 29.3 (1998): 577-598.

David, Charles-Philippe et Stéphane Roussel. "Une espèce en voie de disparition? La politique de puissance moyenne du Canada après la guerre froide." International Journal 52.1 (hiver 1996-1997): 39-68.

Dawspm, Grant. "In support of Peace: Canada, the Brahimi Report and Human Security." Canada among Nations 2001, The Axworthy Legacy. Eds. Fen Olser Hampson, Norman Hillmer et Maureen Appel Molot. Don Mills: Oxford University Press, 2001. 294-317.

Donneur, André P. et Stéphane Roussel. "Le Canada: Quand l'expertise et la crédibilité ne suffisent plus. " Intérêt national et responsabilités internationales. Eds. Alex Macleod et Stéphane Roussel. Montréal: Guérin Universitaire, 1996. 143-160.

Canada. 1994 Le Livre blanc. Ministère de la défense nationale (2002). <http:/ www.forces.gc.ca/admpol/fra/doc/5118_f.htm>.

Canada. Opérations courantes. Ministère de la défense nationale (2004). < http:/ www.forces.gc.ca/site/operations/current_ops_f.asp $>$.

Canada. Politique de défense. Ministère de la défense nationale (2003). <http:/ www.forces.gc.ca/admpol/fra/defence/peace>. 
Canada. Le Canada et le maintien de la paix. Ministère des affaires étrangères et du commerce international (2003). <http://www.maeci-dfait.gc.ca/ peacekeeping/menu-fr.asp $>$.

Jacoby, Tami Amanda. "Canadian peace-building in the Middle East: Case study of the Canada fund in Israel/Palestine and Jordan." Canadian Foreign Policy 8.1 (automne 2000): 83-90.

Jockel, Joseph T. Canada \& International Peacekeeping. Toronto: Canadian Institute of Strategic Studies, 1994.

Rioux, Jean-François et Robin Hay. Canadian foreign policy: from internationalism to isolationism? Ottawa: The Norman Patterson School of International Affairs/Carleton University, 1997.

Schnabel, Albrecht. "L'avenir du maintien de la paix et la viabilité de la sécurité collective: une interprétation réaliste." Tous pour un ou chacun pour soi. Eds. Michel Fortmann, S. Neil MacFarlane et Stéphane Roussel. Québec: Institut québécois des hautes études internationales/Université Laval, 1996. 197-213.

Sokolsky, Joel J. The Americanization of Peacekeeping. Kingston: Center for International Relations/Queen's University, 1997. 